\title{
The Importance of Down Syndrome Phenocopies in the Newborns in Tertiary Obstetric Hospital
}

\author{
Elizabeta Zisovska ${ }^{1^{*}}$, Bratica Lazovska $^{2}$ \\ ${ }^{1}$ Department of Neonatology, University Clinic for Gynecology and Obstetrics, Faculty of Medicine, Skopje, Republic of \\ Macedonia; ${ }^{2}$ Cytogenetic Laboratory, University Clinic for Gynecology and Obstetrics, Faculty of Medicine, Skopje, Republic \\ of Macedonia
}

\begin{abstract}
Citation: Zisovska E, Lazovska B. The Importance of Down Syndrome Phenocopies in the Newborns in Tertiary Obstetric Hospital. OA Maced J Med Sci. 2013 Dec 15; 1(1):32-37. http://dx.doi.org/10.3889/oamjms.2013.007

Key words: dysmorphia; phenocopy; minor anomaly; newborn; Down syndrome.

"Correspondence: Prof. Elizabeta Milan Zisovska. University Clinic for Gynecology and Obstetrics, Neonatology, Vodnjanska 17, Skopje 1000. Republic of Macedonia. Phone: +389 3147 762. E-Mail: zisovska@yahoo.com

Received: 18-Aug-2013; Revised: 14-Sep2013; Accepted: 16-Oct-2013; Online first: 24-Oct-2013

Copyright: () 2013 Zisovska E. This is an open-access article distributed under the terms of the Creative Commons Attribution License, which permits unrestricted use, distribution, and reproduction in any medium, provided the original author and source are credited.

Competing Interests: The authors have declared that no competing interests exist.
\end{abstract}

\begin{abstract}
Background: A phenotype is the composite of the observable characteristics, and in some cases it is not representative for identification of recognized genetic structure.

Aim: The aims of the study were to present the incidence and clinical features of dismorphia in newborn children, and to investigate the prevalence of phenocopies among them.

Material and Methods: Newborns born at the University Clinic for Gynecology \& Obstetrics, having at least 3 minor anomalies $(\mathrm{mm})$ specific for Down syndrome were investigated. Patients' histories, observation, cytogenetic analysis of peripheral blood samples were analysed.

Results: Among 17835 liveborns during 5 years' period, 128 were detected having at least $3 \mathrm{~mm}$, calculated incidence of dysmorphia $0.83 \%$ (1:139). Cytogenetic analysis was not performed in $3.1 \%$ $(4 / 128)$ due to immediate death or transfers elsewhere, $30.5 \%(39 / 128)$ were confirmed Down syndrome. Cytogenetic analysis showed trisomy 21 in $97.4 \%$; Robertsonian translocation had one newborn (2.6\%); normal cytogenetic structure had $66.4 \%(85 / 128)$ of the newborns.

Conclusons: Other studies didn't highlight the proportion of phenocopies of Down syndrome in unselected population of newborns, mainly investigating sick children, disabled, or older-aged. As more the critical role of phenocopy emerges, the more the initial difficulty in detecting gene-gene interactions is amplified. Neglecting the possible presence of phenocopies in complex traits, heavily affects the analysis of their genetic data.
\end{abstract}

\section{Introduction}

A phenotype (from Greek phainein, 'to show, to appear' + typos, 'type') is the composite of an organism's observable characteristics or traits: such as its morphology, development, biochemical or physiological properties, behaviour, and products of behaviour. Phenotypes result from the expression of an organism's genes as well as the influence of environmental factors and the interactions between the two.

The genotype of an organism is a form of inherited instructions carried within genetic code. Not all organisms with the same genotype look or act the same way because appearance and behaviour are modified by environmental and developmental conditions. Likewise, not all organisms that look alike, necessarily have the same genotype [1, 2].
This genotype-phenotype distinction was proposed by Wilhelm Johannsen in 1911 to make the difference clear between an organism's heredity and what that heredity produces $[3,4]$.

The word "phenocopy" was coined by Goldschmit in 1935 [5]. Defined by Free dictionary [6], a phenocopy represents:

1. An environmentally induced phenotype mimicking one, usually produced by a specific genotype, nonhereditary variation in an organism, closely resembling a genetically determined trait;

\section{An individual exhibiting such a variation.}

Because phenocopies may cause problems in genetic screening and genetic counselling, all 
exogenous factors must be ruled out before any congenital trait or defect is labelled hereditary.

It is a generally accepted theory that inherited genotype, epigenetic mechanisms, and non-hereditary environmental variation contribute to the phenotype of an individual [7].

Because a genotype is the mode how the DNA is arranged, and a phenotype is what it shows up as-regardless of the actual genetic code, the phenocopy means that someone has characteristics similar to some genetic combination that the person doesn't possess; it is an imitation of recognized genetic state, generated under the influence of some specific environmental factors. Very often minor anomalies imitate a phenotype of some recognized genetic syndrome, and only cytogenetic analysis may exclude that genetic syndrome [8].

A phenocopy is not a type of mutation; it is an environmentally induced, non-hereditary phenotypic modification that resembles a similar phenotype produced by a gene mutation (genocopy). Some of these features are just traits that are observed to occur more frequently in people with that specific syndrome, but some of the features are more serious medical problems [9].

Minor malformations (minor physical anomalies) are mild physical deformities that with their incidence, number and evolution may be external indicators of hidden, more serious disorders. Minor physical anomalies are defined as unusual morphological features that are found in less than $4 \%$ of the general population and have no serious medical or cosmetic significance to an individual [10]. Most often these are recognized by the neonatologists. First studies done some forty years ago showed an average incidence of $15 \%$ in the general population of newborns and of about $50 \%$ in children with major malformations [11]. Obviously, none of these features is of medical importance, but they are what make people having atypical appearance, making some of the neonatal dysmorphies recognizable [12].

An organism's genotype is the set of genes that it carries. An organism's phenotype is all of its observable characteristics-which are influenced both by its genotype and by the environment. So, in defining evolution, the changes in the genotypes that make up a population from generation to generation are of real concern. However, since an organism's genotype generally affects its phenotype, the phenotypes that make up the population are also likely to change [13]. Aase [14] has referred to them as "microsigns." Usually these are morphogenetic disturbances settled between normal variations and expressed morphological changes [15].

Before giving importance to a separate morphological feature, it should be necessary to observe the members of the close family, because such a common sign identified in the healthy family members within the certain family is considered as "marker" of inherited disturbance of the morphogenesis [16-19]. The value of the early recognition of the minor malformations is their ability to lead towards investigation for a major anomaly (pathology of the morphogenesis) in common, or to give a suspicion for a major anomaly or genetic syndrome. It is worth mentioning that not only the number of minor malformations is important, but also their scoring weight, actually the degree upon which the anatomical structure deviates from the normal [20]. When several specific minor malformations are seen together in an individual, they can serve as external markers for underlying genetic disorders [21]. It is of importance to present the correlation between the number of the minor anomalies and the risk for presence of major anomaly found in different sources of information: if the individual doesn't have any minor anomaly, the probability of existence of major anomaly is about 1.4\%; one minor malformation carries a probability for major anomaly of $3 \%$; if two minor malformations are present in the same person, the probability of existing major anomaly is about 11$15 \%$, and if three or more minor anomalies are present, the probability of major anomaly, especially chromosomal abnormality, raises over 50\% [22, 23]. Less than $1 \%$ of newborns have three or more anomalies, and these newborns are at higher than $20 \%$ risk for a major malformation [24, 25]. Another source of information presents that children who have two minor anomalies have a $10 \%$ risk of developing major anomalies, finally, those children with 3 or more minor anomalies have again a $20 \%$ risk of developing a major anomaly [26]. The incidence of minor anomalies has been described between $7 \%$ and $41 \%$, while the incidence of major anomalies has been described between $2 \%$ and $3 \%$ [23, 27]. Therefore, the presence of a minor anomaly should alert the medical professional for the possibility of major defect, whereas the existence of an anatomical variation has not the same meaning. The causes of the variations and anomalies must be found in the biologic processes of development during the formation of a particular structure; this may be due to genetic reasons, environmental factors or combinations amongst them.

The clinical features that characterize the phenotype of the Down syndrome, represent the most frequent chromosomal abnormality. Those newborns/infants have well recognized characteristic appearance, although varying in the combination of minor anomalies. In 1866, Down described 21 specific physical anomalies that included small ears, upslanting palpebral fissures, flat nasal bridge, epicanthal folds, protruding tongue, small mouth, fifth finger clinodactyly and a wide space between the first and second toes that characterized a type of mental retardation that he called "mongolism," which today is known as Down syndrome [28]. The inner corner of the eyes may have a rounded fold of skin (epicanthal fold). The hands are short and broad with short 
fingers, and may have a single palmary crease. White spots on the colored part of the eye called Brushfield spots may be present. Babies with Down syndrome often have decreased muscle tone at birth. Normal growth and development is usually delayed and often individuals with Down syndrome don't reach the average height or developmental milestones of unaffected individuals. It is important to remember that no one having Down syndrome will have all of the features described above, and not all of the features described are of importance for the health condition of the patient. Nor does the number of physical problems in a person with Down syndrome correlate with their intellectual capability. Each and every child with Down syndrome has their own unique personality and strengths.

The purpose of listing these features is to give information about the wide range of variability in minor malformations that can be seen in newborns with Down syndrome, thus implying that none of these malformations alone is of vital, medical or social importance for the person [29, 30]. The existence of phenocopy may cause problem in determining the exact diagnosis of congenital abnormalities in newborn children, especially chromosomal abnormalities, and may cause psychological and emotional stress for the parents and difficulties for counsellors and physicians in managing this medical issue. study was to:

Thus, the purpose of this prospective 5 year's

- record the incidence of dysmorphia (minor malformations) for Down's syndrome in newborn children successively born at the University Clinic for Gynaecology and Obstetrics in Skopje, Republic of Macedonia;

- determine the top ten minor dysmorphic abnormalities for Down's syndrome in the investigated population;

- analyze the cytogenetic structure (abnormalities) of these dysmorphic children and investigate the proportion of the phenocopies among these dysmorphic newborns.

\section{Material and Methods}

In this study we have investigated 17835 newborns, succesively born at the University Clinic for Gynecology and Obstetrics, Skopje, Republic of Macedonia, in the period of five years (2008-2012). The study was prospective, observational by its design. The Clinic provides tertiary level of care, thus admitting all high risk pregnant women for further care and treatment. During the study, several analytical processes were performed: history data obtained from the maternal medical records; observation and diagnosis of dysmorphia of the newborn; classification of the minor anomaly; assessing the indication for cytogenetic analysis; simple statistical measures of retrieved data.

As minor anomalies were considered all those which have no functional impact on the neonatal health, according to the accepted list of minor anomalies, classified as shown in Table 1 [11, 30].

Table 1: The List of the most frequent minor malformations.

\begin{tabular}{l} 
Localization and type of the most common referred and described minor \\
anomalies \\
\hline Head and neck: brachycephaly, megalencephaly, microcephaly, fine, electric hair, \\
vowleak hair, aberrant hair whorls pattern (two or more), upward or downward slanting \\
eyes, epicanthal fold, hypertelorism, white spots on the coloured part of the eye, \\
synophrus, flattened nose (flat nasal bridge), bulbous nasal tip, bifid nasal tip, \\
anteverted nares, smooth filtrum, ear tags, malformed ears (auricula) by any means, \\
adherent lobes of the auricula, thin upper limb, epithelial cysts of the alveolas, \\
retrognathia and microgenia, high and oval palate, protruding tongue and open mouth, \\
premature dentition, tongue tie, lingua geographica, bifid uvula, grooved chin, \\
pterygium, short and wide neck, low hair on the neck. \\
\hline Chest and Abdomen: Lateral position of the nipples, low position of the umbilicus. \\
\hline Arms and legs: four finger line of the palms, short, broad palms and short fingers, \\
oedema on the hands, clinodactily of the fifth finger, wide and short thumb, backwards \\
pointing thumb, partial syndactily of the third and fourth finger of the hand, bent index \\
finger, tapered fingers, short toes, wide hallux, third leg finger equal or longer than the \\
second, second toe linger than the first, wide distance between the first and second toe \\
("sandal gap"), overlapping toes, hammertoes, deep plantar crease. \\
\hline General signs: decreased muscle tone (hypotonia), excessive joint laxity, \\
expressionless face, dry skin.
\end{tabular}

Other indications for cytogenetic analysis that were not part of this study were:

- Existence of familiar genetic disease, chromosomopathy or X-linked diseases;

- Prenatal suspicion of chromosomal anomaly in pregnancy;

- Undetermined sex after the delivery;

- Association of two or more major congenital anomalies.

\section{Results}

In the study 128 newborn children were analyzed selected on the basis of the number of 51 minor anomalies, using as inclusion criteria presence of at least 3 minor anomalies (dysmorphic features) characteristic for Down syndrome. The number of live births during the investigated period was 17835, so the incidence of highly dysmorphic newborns was $128 / 17835$, i.e. $1 / 139(0.83 \%)$ in the population of liveborn newborns. The ten most frequent minor anomalies imitating features for Down syndrome are presented in Table 2.

All of the 128 selected infants had an indication to perform cytogenetic analysis in peripheral blood cells, to determine their karyotype. The analysis showed following results (Fig 1 ): $3.1 \%(4 / 128)$, equal number (2:2) of both sexes, were not analyzed due to immediate death, or transfer to other Clinics because of different diseases; $30.5 \%$ (39/128) were cytogenetically confirmed as Down syndrome, and 
fortunately, $66.4 \%(85 / 128)$ had normal karyotype, i.e. were phenocopies of Down syndrome.

Table 2: Top ten minor malformations in newborns suspected for Down syndrome.

\begin{tabular}{lc}
\hline Minor malformation & \% of investigated \\
\hline Decreased muscle tone & 95 \\
Flat nasal bridge & 92 \\
Upward slanted eyes & 92 \\
Hypertelorism & 90 \\
Tongue protrusion & 83 \\
Low hair line on the neck & 82 \\
Epicanthal folds & 82 \\
Swollen, wide and short palms & 78 \\
Unilateral simian crease & 74 \\
Excessive joint laxity & 75 \\
\hline
\end{tabular}

There was striking difference between male and female newborns in this subgroup, actually the ratio male:female was 25:60 in favour of the females.

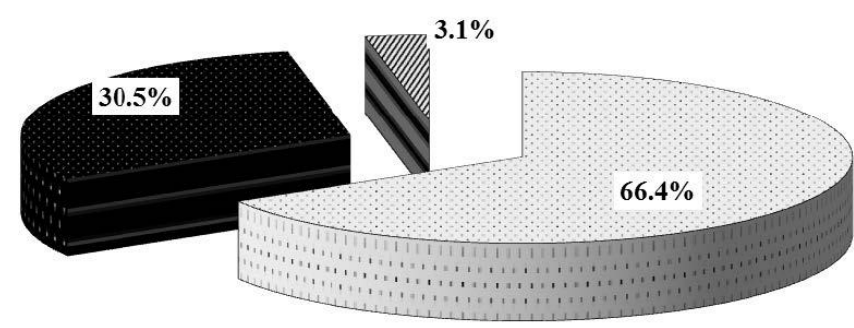

[normal karyotype Down syndrome $\square$ not analyzed

Figure 1: Cytogenetic findings at the dysmorphic newborns with Down sy stigmata.

Cytogenetic detailed analysis of the peripheral blood cells of the patients with confirmed Down syndrome showed trisomy 21 in 38/39 (97.4\%), structure $47, X X,+21$ or $47, X Y,+21$; and $1 / 39(2.6 \%)$ Robertsonian translocation 46, $X Y$, rob $(14 ; 21)$ (q10;q10), +21 (Fig 2).

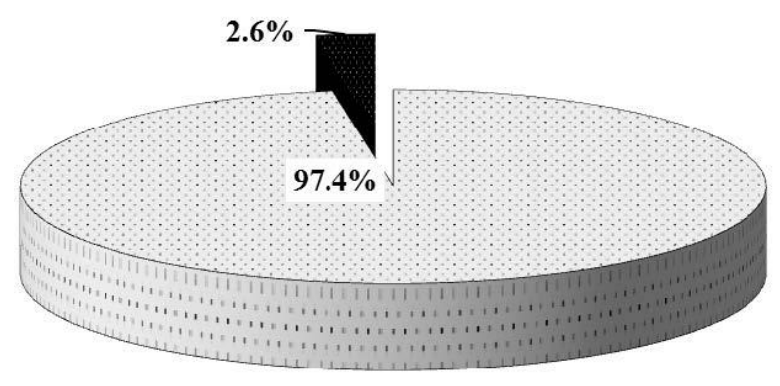

$\square$ trisomia 21

a translocation

Figure 2: Proportional relationship of the chromosomal structure in newborns with Down syndrome.

There was found weak difference between male and female newborns who had confirmed Down syndrome, in favor of the males, 22/39 (56.4\%). The overall ratio male:female is presented in Fig. 3.

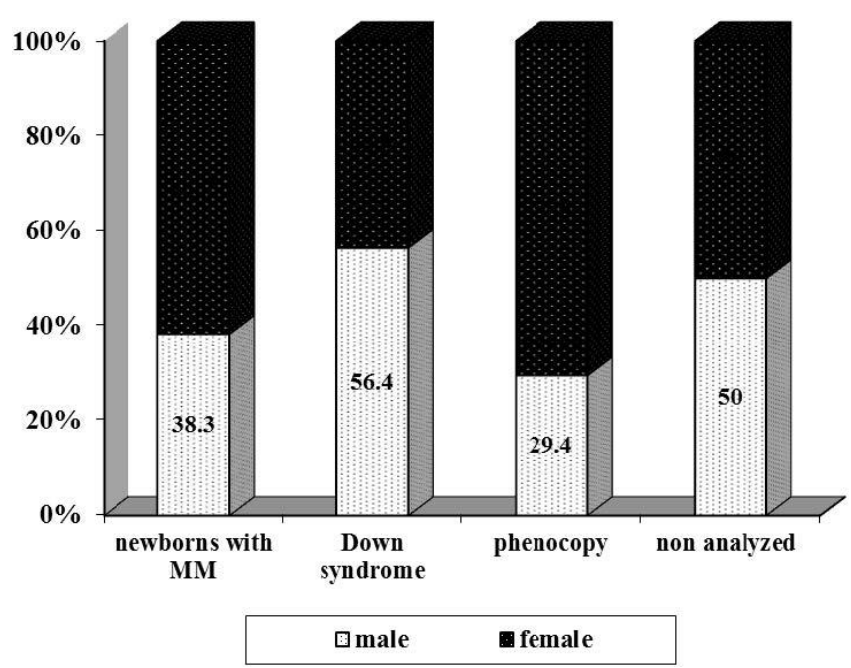

Figure 3: The overall ratio male:female in dysmorphic newborns.

Positive predictive value of the finding of at least three minor anomalies for predicting major anomaly is $31.45 \%$. Investigating the closest members of the families of the newborns having at least three minor anomalies, in 22/139 cases (15.8\%) at least one of the presented minor malformations were found in another family member. Only 3 cases of confirmed Down syndrome belonged to a family having any of the minor malformations before mentioned as a "marker".

\section{Discussion}

Deep search within the literature has not resulted in retrieving papers about the proportion of phenocopies in unselected population of newborn infants. It was usually investigated in sick children, children with disabilities, or older aged children [3135]. So, our results should be considered as rare attempt to analyze the possibility of existing phenocopies, thus helping in the differentiation of chromosomal abnormalities of any kind.

In literature it was reported that overall incidence of minor anomalies among unselected neonatal population is $5.03 \%(55.55 \%$ male and $44.46 \%$ female, $p>0.05$ ), and $0.28 \%$ of neonates had at least two minor anomalies [36]. Another study has shown different results, considering different type of population (Chinese), whereas some of the variations/minor anomalies are met as normal variations [37]. About $44.9 \%$ of the newborn infants had at least one minor anomaly that was unrelated to gender, maternal age, or gestational age, but significantly associated with fetal presentation. Breech-presented newborn infants had double the risk of minor anomalies. Simian crease, upward slant and frontal bossing could be considered normal variants 
for Chinese newborn infants, because the incidence of each was higher than $4 \%$. Although some studies have shown that approximately $90 \%$ of infants having three or more minor anomalies are associated with a major malformation, there was found pretty low, $31.45 \%$ predictive value based on these criteria. Nevertheless, it was suggested that infants with three or more minor anomalies should be carefully evaluated for the possibility of major malformation in order to provide early management [37].

There was low proportion of translocation among the newborns with confirmed Down syndrome, only $2.6 \%$. Interesting results were in terms of the ratio male:female. In the group of Down syndrome, male prevailed over the female infants, while in the group of phenocopies, striking difference was found in favor of the females. This fact could be interpreted that the girls have more dysmorphia-like features than real minor malformations.

The list of top ten presented minor malformations has shown that $\geq 90 \%$ of the newborns had decreased muscle tone, flat nasal bridge, upward slanted eyes, and hypertelorism. These signs should be taken as crucial for considering the child as potential Down syndrome.

The key point is the capability to identify lowpenetrance variations across the human genome. Among the phenomena reducing the power of these analyses, phenocopy level hampers very seriously the investigation of complex diseases, as well known in neurological disorders, cancer, and likely of primary importance in human ageing. Phenocopy level seems to be the norm, rather than the exception, especially when considering the role of epigenetic and environmental factors towards phenotype [38].

The signs and features of the Down syndrome are result of slower process of maturation of the brain and the body until fetal period, and incomplete morphogenesis. During the intrauterine period the obstetrician has to possess very high level of skills, knowledge and ability to employ modern technology to recognize the subtle signs of dysmorphy [39]. The dysmorphic newborns could have various ranges of minor malformations, some of them with subtle expression. The fact that the combinations are unpredictable and are non-standard makes more difficult their recognition. Without cytogenetic analysis, fenocopies can extend the problem regarding the confirmation of the predicted diagnosis, and create anxiety among parents.

The usefulness and importance of the cytogenetic analysis is higher when it is performed even if suspected chromosomal anomaly, because it would be confirmed very early, and the completeness of the working out should be achieved sooner, thus enabling appropriate follow up and stimulation.

Our results have suggested the following:
- The detailed observation looking for the subtle dysmorphic signs could be very important an useful;

- Phenocopies are very common in the states of neonatal dysmorphy;

- Phenocopies aggravate the clinical diagnosis of the chromosomal abnormalities;

- The confirmation of phenocopy is the most desirable diagnosis in dysmorphic child.

As a summary, it could be recognized that certain facts call for further analysis, the evidence which has thus far been brought forward from various sources justifies the following interpretation of the genesis of phenocopies. The point of interference varies according to the existing genotype, the developmental stage, kind and quantity of the external agent, and other factors. Taking all evidence into account, it seems that the most exciting aspect of the study of phenocopies is the opportunity it may provide of shedding light, although only indirectly, on the developmental functions of that awesome skeleton of genetical science-the normal genotype.

The complexity of contigent relationsrelations between genotype and phenotype arise from the nature of organisms as physical systems. As is true for living systems in general, relations between genotype and phenotype are contingent, varying from case to case. Still, the critical role of phenocopy emerges, and the more the phenocopy level increases, the more the initial difficulty in detecting gene-gene interactions is amplified.

It could be concluded that the neglect of the possible presence of phenocopies in complex traits, heavily affects the analysis of their genetic data. According to our research it is recommended to do cytogenetical analyses of all newborns with phenocopy of Down's syndrome.

\section{References}

1. Jablonka E. Current Biology: Transgenerational epigenetic inheritance: prevalence, mechanisms, and implications for the study of heredity and evolution The Quarterly Review of Biology. 2009;84(2): 131-176.

2. Edwards AW. Human genetic diversity: Lewontin's fallacy. Bio Essays 2003; 25 (8): 798-39 801

3. Roll-Hansen, Nils. Sources of Wilhelm Johannsen's Genotype Theory. Journal of the History of Biology. 2009; 42 (3): 457-93.

4. Lewontin R. The Genotype/Phenotype Distinction, The Stanford Encyclopedia of Philosophy (Summer 2011 Edition), Edward N.

Zalta (ed.), URL

http://plato.stanford.edu/archives/sum2011/entries/genotypephenotype/

5. Widukund L. Springer-Werlag Humangenetik. 1970; 9:227-229.

6. http://medical-dictionary.thefreedictionary.com/ accessed 2013 08-18. 
7. Landauer W. On phenocopies, their developmental physiology and genetic meaning. American naturalist. 1958; 92(865): 210-215.

8. Relethford JH. Apportionment of global human genetic diversity based on craniometrics and skin color. Am J Phys Anthropol. 2002 118 (4): 393-8.

9. Nachtomy $O$, et al. Gene expression and the concept of the phenotype. Studies in History and Philosophy of Biological and Biomedical Science. 2007; 38 (1): 238-54.

10. Jones K. Smith's Recognizable Patterns of Human Malformation. Vol. 4th. WB Saunders Co.: Philadelphia, 1988.

11. Sumanović-Glamuzina D, Bozić T, Brkić V, Robović A, SaragaKaracić V. Minor malformations: neonatal or anthropological story? Coll Antropol. 2009; 33(Suppl 2):31-5

12. McAdams $\mathrm{H}$, Arkin A. Stochastic Mechanisms in Gene Expression. Proceedings of 10 the National Academy of Sciences, U.S.A., 1997; 94: 814-819.

13. Levy S, Sutton G, Ng PC, Feuk L, Halpern AL, et al. The Diploid Genome Sequence of an Individual Human. PLoS Biol. 2007; 5 (10): e254.

14. Aase J. Diagnostic Dysmorphology; Plenum, New York, 1990.

15. Lerer RJ. Do hyperactive children tend to have abnormal palmar creases? A report of a 16 suggestive association. Clin Pediatr. 1977;16:645-647.

16. Manica A, Amos W, Balloux F, Hanihara T. The effect of ancient population bottlenecks on human phenotypic variation. Nature. 2007;448 (7151): 346-8.

17. Lee M. Ethnicity tied to gene expression - The Scientist Magazine of the Life Sciences. The Scientist. Accessed 2013-01 25.

18. Common genetic variants account for differences in gene expression among ethnic groups: Abstract: Nature Genetics. Nature.com. Accessed 2013-02-15.

19. Reich D, Green RE, Kircher M. Genetic history of an archaic hominin group from Denisova Cave in Siberia. Nature. 2010; 468 (7327): 1053-1060.

20. Waldrop M, Halverson C. Minor physical anomalies and hyperactive behavior in children. In: Hellmuth $\mathrm{J}$ (ed) Exceptional Infant: Studies in Abnormalities. Brunner/Mazel: New York, 1971;(2):343-380.

21. Hoyme H. Minor anomalies: diagnostic clues to aberrant human morphogenesis. Genetica. 1993; 89:307-315.

22. [Zisovska E. The role of minor anomalies in the early childhood in early recognition of hereditary anomalies and chromozomopathies. [MSc] UKIM: Skopje, 1983]. In Macedonian.

23. Fontaine $\mathrm{CH}$. Some thoughts about anatomic variations. Surg Radiol Anat. 2001;23: 1-2.

24. Marden PM, Smith DW, MC Donald MJ. Congenital anomalies in the newborn infant, including minor variants. J Paediatrics. 1964; 64: 357-371.

25. Stevenson RE, Hall JG. Terminology. Vol I. In: Stevenson RE, Hall JG and Goodman RM (eds). Human malformations and related anomalies. Oxford University Press: London, 1993; pp 21-30.

26. Mehes K, Stadler G. Minor malformations in the neonate. Akadémiai Kiadó: Budapest, 1983.

27. William PLT, Humpherson JR. Concepts of variation and normality in morphology: import issues at risk of neglect in modern undergraduate medical courses. Clin Anat. 1999; 4912: 186-190.

28. Arey LB. Developmental Anatomy. W.B. Saunders Company: Philadelphia and London, 1940.

29. Down J. Observations on an ethnic classification of idiots. London Hospital Clinical Lecture Reports. 1866; 3:259-262.
30. Pinsky L. Informative morphogenetic variants: minor congenital anomalies revisited. In: Kalter $\mathrm{H}$, ed. Issues and Reviews in Teratology. Plenum Press: New York, 1985;3:135-588.

31. Accardo PJ, Tomazic T, Morrow J, Haake C, Whitman BY. Minor Malformations, Hyperactivity, and Learning Disabilities. Arch Pediatr Adolesc Med. 1991;145(10):1184-1187.

32. Hoyme HE. Minor malformations: significant or insignificant? AJDC. 1987;141:947.

33. Green MF, Satz P, Soper HV, Kharaki F. Relationship between physical anomalies and age of onset of schizophrenia. Am J Psychiatry. 1987;144:666-667.

34. Miller G. Minor congenital anomalies and ataxic cerebral palsy . Arch Dis Child. 1989;64:557-562.

35. Drillien C, Drummond M. Developmental Screening and the Child with Special Needs. Philadelphia, Pa: JB Lippincott, 1983.

36. Pouladfar G, Mallahzadeh A. The Prevalence of minor congenital anomalies and normal variations in neonates in Bushehr port. 2005; 8(1): 43-52.

37. Tsai FJ, Tsai $\mathrm{CH}$, Peng $\mathrm{CT}$, Wu JY, Lien $\mathrm{CH}$, Wang TR. Different race, different face: minor anomalies in Chinese newborn infants. Acta Paediatr. 1999; 88(3):323-6.

38. Lescai F, Franceschi C. The Impact of Phenocopy on the Genetic Analysis of Complex Traits. PLoS ONE. 2010; 5(7): e11876.

39. Trent RJA, Williams R and Sutherland GR. The "new" genetics and clinical practice. MJA. 2003; 178: 406-409. 